\title{
MEDICION DE LOS NIVELES DE RUIDO EN LA CIUDAD DE HUACHO-2014
}

\author{
MEASUREMENT OF NOISE LEVELS IN THE CITY OF HUACHO -2014 \\ José Luis, Romero Bozzetta ${ }^{1}$, Luis Alberto, Huayna Dueñas ${ }^{1}$, William Guzmán Sancheza ; \\ Adelfio Bacilio Alvarado', Oscar Torres Mejia
}

\section{RESUMEN}

El objetivo fue determinar qué zonas de la ciudad de Huacho, son las que tiene mayor contaminación auditiva; provocada por el parque automotor, con la finalidad de implementar estrategias para disminuir los decibeles causantes de tal contaminación, y así mejorar la salud de los habitantes y los marchantes de la zona. En el procedimiento empleado se tomaron 5 puntos de muestreo, escogidas por la mayor afluencia de público y por la cantidad de vehículos de combustión. Se aplicaron encuestas y se midió la cantidad de decibeles en las zonas escogidas. Los resultados indicaron que, El 70 \% de los encuestados opina que el lugar más ruidoso es el óvalo. Del mismo modo, el $60 \%$ opinan que el lugar con ruido intermedio es 28 de julio; y, $50 \%$ consideran que el lugar menos ruidoso es la calle Grau. Se indica además que la zona de mayor ruido en la mañana y tarde es el Ovalo de Huacho, y las de menor ruido son en las afueras de la Universidad José Faustino Sánchez Carrión en las mañanas, tan igual como afuera del colegio San Ignacio de Loyola turno de mañana. En conclusión, se determinó que la zona de mayor ruido es el ovalo de Huacho.

Palabras clave: Contaminación auditiva; Decibeles; Sonómetro

\begin{abstract}
The objective was to determine which parts of the city of Huacho, are those who have greater noise pollution; caused by the vehicle fleet, in order to implement strategies to reduce decibels causes of such pollution, and improve the health of residents and dealers in the area. 5-point sampling, chosen by the largest number of visitors and the amount of combustion vehicles were taken. Surveys were applied and the amount of decibels measured in targeted areas. The results indicated that $70 \%$ of respondents believe that the loudest place is the oval. Similarly, $60 \%$ think that the intermediate noise place July 28; and $50 \%$ believe the place is quieter street Grau. It also indicates that the area of greatest noise in the morning and evening is the oval of Huacho, and lower noise are outside of the University José Faustino Sánchez Carrión in the mornings, as equal as outside the College San Ignacio de Loyola morning shift. Conclusion was determined that the area of greatest noise is the oval of Huacho.
\end{abstract}

Key words: Noise pollution; Decibel; Sound level meter.

$\overline{1}$ Universidad Nacional José Faustino Sánchez Carrión de Lima (Lima - Perú). Email:vinvestigación@unjfsc.edu.pe 


\section{INTRODUCCIÓN}

El oído humano es muy sensible a las fluctuaciones de presión del aire. La percepción sensorial de este fenómeno es lo que llamamos sonido. Dichas vibraciones del aire se propagan en forma ondulatoria desde la fuente de sonido, cuando la fuente deja de vibrar, el sonido se detiene. Llamamos ruido a un sonido no deseado o molesto. La clasificación de un sonido en la categoría de ruido es subjetiva, dependiendo de los individuos y las circunstancias.

Las tres principales características del sonido son: amplitud, frecuencia y patrón temporal.

La amplitud es percibida como la fuerza o sonoridad del sonido. Se la cuantifica a través del Nivel de Presión Sonora, expresado en decibeles. El valor mínimo que puede percibir el oído humano es de 0 dB. Al valor máximo se lo denomina umbral de dolor y es de unos $130 \mathrm{~dB}$. El ruido comienza a dañar la audición aproximadamente a los $70 \mathrm{db}$.

La OMS considera que el límite superior deseable es de $50 \mathrm{~dB}$, mientras que España ubica el nivel de confort acústico en los $55 \mathrm{~dB}$.

En los últimos años la contaminación acústica (CA) se ha extendido en el tiempo (circulación nocturna, fines de semana, vacaciones, etc.) y en el espacio (zonas rurales y zonas residenciales de las grandes ciudades) debido, en gran medida, al aumento del tráfico rodado y del aéreo y al incremento de la densidad de población. Consecuentemente, las áreas libres de CA ocasionadas por el tráfico, la industria o la agricultura, se han hecho cada vez más escasas a escala europea y la tranquilidad se percibe como un recurso cada vez más valioso. (García 1996). De este modo, áreas hasta el momento exento de este tipo de contaminación, tales como las masas forestales bien conservadas del territorio, han pasado a verse notablemente afectadas.

Existen varios mecanismos de exposición a un ambiente ruidoso, puede ser de manera continua, fluctuante, intermitente o impulsiva, y de ello dependerá la profundidad y la rapidez con que se desarrolle la pérdida auditiva, aunque en cualquiera de los casos, es lamentablemente irreversible. (Ramírez 1998)

Por todos es conocido el significado de la audición en el humano para la comunicación y la interrelación con el medio que nos rodea; por lo que el deterioro auditivo hace de las personas, un ser retraído, desconfiado y expuesto a
peligros.(Eriksson-Mangold y Carisson 1991), refieren que las pérdidas auditivas pueden generar inseguridad en situaciones en que la persona debe desenvolverse en la sociedad, conduciéndolo a una disminución de la sensación de control y en consecuencia se puede producir un estrés psíquico y físico, que podría llevar al sujeto a la depresión.

Portman, Álvarez y otros autores, definen la hipoacusia inducida por ruidos como la disminución de la capacidad auditiva de uno o de ambos oídos, parcial o total, permanente o acumulativa, de tipo sensorineural, que se origina durante y como resultado de la exposición a niveles perjudiciales de ruido en el ambiente laboral; esta clase de hipoacusia se corresponde mayormente con el trauma acústico.(Portman 1997) (Bosshardt 1995)

Morales y Correa, definen el trauma acústico como "toda lesión producida en el oído interno, ocasionada por exposición a ruidos". Estos autores hacen una diferenciación teniendo en cuenta la duración e intensidad del ruido, y de acuerdo a ello emiten dos categorías conceptuales (Portman 1997), que se corresponden también con las emitidas por López AC. y cols. (López 2000):

Trauma acústico: Pérdida auditiva secundaria a la exposición a un ruido único y de gran intensidad (explosión, tiro, etc).

Deterioro auditivo inducido por ruidos: disminución progresiva y gradual de la agudeza auditiva, ocasionada por la exposición prolongada a ruidos de moderada intensidad.

El presente estudio, se justifica porque, este tipo de contaminación puede perfectamente ser medida y evaluada. Siendo conocidas las consecuencias que acarrea para la salud física y mental de las personas que se exponen a ella, Conociendo las actividades que promueven la contaminación sonora o auditiva tales como fábricas e industrias, las actividades relacionadas con la construcción, el tráfico y bocinas, locales de ocio (discotecas, Pubs, boliches, lugares de juego de video, etc), aviones, trenes, los gritos, por nombrar los más comunes, que producen efectos negativos sobre la salud auditiva física y mental de las personas; será la base para implementar las estrategias de control de ruidos molestos.(Álvarez 1967)

\section{MATERIALY MÉTODOS}

\section{1 Área de estudio:}

Se estudiará las zonas con mayor afluencia de 
público y concentración de vehículos automotores

\subsection{Población:}

La población estará conformada por los habitantes y parque automotor existente en la ciudad de Huacho.

\subsection{Muestra:}

Se tomarán 5 puntos de muestreo, escogidas por la mayor afluencia de público y por la cantidad de vehículos de combustión.

\subsection{Materiales, Equipos y Recursos humanos}

\subsubsection{Materiales}

- Lápices

- Tableros de madera

\subsubsection{Equipos}

\section{- Sonómetro}

- Cámara Digital de 24 pixeles.

\subsubsection{Otros}

- Mapas de la zona de trabajo

\subsection{Estrategia de muestreo:}

Una vez han quedado definidos los parámetros que se han de reflejar en el plano acústico del ambiente, en función de las necesidades de información y de la disponibilidad de instrumentación y recursos humanos para realizarlo, queda por determinar, como parámetros más importantes y que dependen de la naturaleza de las fuentes sonoras y de la topología del ambiente, las pautas del análisis estadístico a aplicar, tanto temporal como espacial. Existen dos estrategias metodológicas para la toma de medidas, denominadas metodología estática y metodología dinámica. Ambas estrategias realizan un muestreo espacial (distintos puntos de medidas y su muestreo temporal, y ciclos de evolución del ruido), diferenciándose en la forma de hacerlo

Se diseñó la forma de evaluar el impacto acústico en 5 vías de tráfico, colocándose en la esquina de todas las zonas de estudio.

- Se recopiló información de trabajos similares realizados a nivel Nacional e internacional.

- También se confecciona el instrumento encuesta donde se les realizó una entrevista abierta a los habitantes marchantes.

\subsection{Técnicas de recolección de datos}

Se ubicaron los 5 puntos de mayor afluencia de público y de mayor numero de vehículos automotores, donde se procedió a tomar las medidas acústicas con el sonómetro y aplicar encuestas a las personas que se encontraban en la zona.

2.7 Técnicas para el procesamiento de la información

Los resultados obtenidos se tabularán en correspondencia a los objetivos propuestos para el estudio. Se acompañaran cuadros, gráficos y otras a fin de facilitar el análisis e interpretación de los resultados para lo cual se utilizará un programa estadístico SPSS 17 utilizando una computadora Pentium dual core con software Word for Windows, Excel, para el análisis estadístico.

También para el análisis estadístico se usó la prueba de tukey, considerando un $\mathrm{p} \leq 0.05$ como nivel de significancia.

\section{RESULTADOS}

Tabla 1. Frecuencias (db) de respuesta de los encuestados para el ítem 10: Lugar más ruidoso

\begin{tabular}{llcc}
\hline OPCION & LUGARES & FRECUENCIA & PORCENTAJE \\
\hline a & Óvalo & 35 & 70.00 \\
& 28 de julio & 10 & 20.00 \\
& San Martín & 5 & 10.00 \\
$\mathrm{~b}$ & 28 de julio & 30 & 60.00 \\
& Grau & 15 & 30.00 \\
& San Martín & 5 & 10.00 \\
$\mathrm{c}$ & Grau & 25 & 50.00 \\
& Echenique & 5 & 10.00 \\
& 28 de julio & 5 & 10.00 \\
& Leoncio Prado & 10 & 20.00 \\
& San Martín & 5 & 10.00 \\
\hline
\end{tabular}

El $70 \%$ de los encuestados opina que el lugar más ruidoso es el óvalo. Del mismo modo, el $60 \%$ opinan que el lugar con ruido intermedio es 28 de julio; $y, 50$ $\%$ consideran que el lugar menos ruidoso es la calle Grau. 
Tabla 2. Frecuencias de respuestas de los encuestados con respecto al lugar más ruidoso

\begin{tabular}{|c|c|c|c|c|c|c|c|c|c|c|c|c|c|c|c|}
\hline \multirow[t]{2}{*}{$\mathrm{N}^{\circ}$} & \multicolumn{3}{|c|}{$\begin{array}{c}\text { Fuera del Colegio San } \\
\text { Ignacio de Loyola }\end{array}$} & \multicolumn{3}{|c|}{ Óvalo } & \multicolumn{3}{|c|}{28 de julio con Echenique } & \multicolumn{3}{|c|}{ San Martín } & \multicolumn{3}{|c|}{ Fuera de la UNJFSC } \\
\hline & Mañana & Tarde & Total & Mañana & Tarde & Total & Mañana & Tarde & Total & Mañana & Tarde & Total & Mañana & Tarde & Total \\
\hline 1 & 28 & 18 & 46 & 30 & 35 & 65 & 20 & 28 & 48 & 25 & 36 & 61 & 26 & 25 & 51 \\
\hline 2 & 19 & 21 & 40 & 15 & 22 & 37 & 26 & 21 & 47 & 23 & 13 & 36 & 20 & 22 & 42 \\
\hline 3 & 3 & 1 & 4 & 0 & 0 & 0 & 4 & 0 & 4 & 2 & 1 & 3 & 4 & 3 & 7 \\
\hline 4 & 25 & 19 & 44 & 27 & 30 & 57 & 36 & 32 & 68 & 29 & 36 & 65 & 19 & 23 & 42 \\
\hline 5 & 17 & 13 & 30 & 17 & 15 & 32 & 3 & 16 & 19 & 6 & 4 & 10 & 17 & 15 & 32 \\
\hline 6 & 5 & 8 & 13 & 5 & 5 & 10 & 11 & 2 & 13 & 15 & 10 & 25 & 14 & 12 & 26 \\
\hline 7 & 22 & 15 & 37 & 17 & 14 & 31 & 3 & 9 & 12 & 19 & 3 & 22 & 7 & 15 & 22 \\
\hline 8 & 22 & 20 & 42 & 29 & 31 & 60 & 33 & 26 & 59 & 15 & 32 & 47 & 28 & 22 & 50 \\
\hline 9 & 6 & 5 & 11 & 4 & 5 & 9 & 14 & 15 & 29 & 16 & 15 & 31 & 15 & 13 & 28 \\
\hline 10 & 23 & 21 & 44 & 22 & 17 & 39 & 21 & 18 & 39 & 20 & 38 & 58 & 22 & 18 & 40 \\
\hline 11 & 23 & 15 & 38 & 20 & 19 & 39 & 16 & 14 & 30 & 12 & 9 & 21 & 18 & 20 & 38 \\
\hline 12 & 4 & 4 & 8 & 8 & 10 & 18 & 13 & 18 & 31 & 18 & 3 & 21 & 10 & 11 & 21 \\
\hline 13 & 21 & 13 & 34 & 11 & 11 & 22 & 24 & 22 & 46 & 38 & 22 & 60 & 19 & 20 & 39 \\
\hline 14 & 16 & 15 & 31 & 24 & 23 & 47 & 20 & 18 & 38 & 11 & 14 & 25 & 18 & 24 & 42 \\
\hline 15 & 13 & 12 & 25 & 15 & 16 & 31 & 6 & 10 & 16 & 1 & 14 & 15 & 13 & 16 & 29 \\
\hline 16 & 18 & 21 & 39 & 30 & 20 & 50 & 18 & 28 & 46 & 12 & 17 & 29 & 20 & 12 & 32 \\
\hline 17 & 22 & 11 & 33 & 20 & 20 & 40 & 21 & 16 & 37 & 18 & 17 & 35 & 22 & 22 & 44 \\
\hline 18 & 10 & 8 & 18 & 0 & 0 & 0 & 11 & 6 & 17 & 20 & 13 & 33 & 8 & 16 & 24 \\
\hline 19 & 29 & 14 & 43 & 28 & 25 & 53 & 46 & 36 & 82 & 44 & 45 & 89 & 23 & 34 & 57 \\
\hline 20 & 21 & 26 & 47 & 5 & 3 & 8 & 4 & 13 & 17 & 6 & 5 & 11 & 24 & 11 & 35 \\
\hline 21 & 0 & 0 & 0 & 3 & 3 & 6 & 0 & 0 & 0 & 0 & 0 & 0 & 3 & 4 & 7 \\
\hline 22 & 23 & 16 & 39 & 25 & 22 & 47 & 27 & 34 & 61 & 42 & 34 & 76 & 26 & 27 & 53 \\
\hline 23 & 20 & 21 & 41 & 7 & 5 & 12 & 11 & 11 & 22 & 2 & 11 & 13 & 12 & 17 & 29 \\
\hline 24 & 7 & 3 & 10 & 5 & 4 & 9 & 5 & 3 & 8 & 6 & 5 & 11 & 4 & 6 & 10 \\
\hline 25 & 20 & 16 & 36 & 14 & 17 & 31 & 16 & 22 & 38 & 29 & 23 & 52 & 21 & 16 & 37 \\
\hline 26 & 20 & 20 & 40 & 28 & 25 & 53 & 30 & 23 & 53 & 18 & 25 & 43 & 23 & 27 & 50 \\
\hline 27 & 10 & 4 & 14 & 8 & 8 & 16 & 4 & 3 & 7 & 3 & 1 & 4 & 6 & 7 & 13 \\
\hline
\end{tabular}

Tabla 3. Test de homogeneidad de promedios La prueba de homogeneidad de promedios señala

\begin{tabular}{|c|c|c|c|}
\hline VARIA & MEDIA & \multirow{2}{*}{\multicolumn{2}{|c|}{$\begin{array}{c}\text { HOMOGENEIDAD } \\
\text { A }\end{array}$}} \\
\hline T1 & 13.33 & & \\
\hline T2 & 15.00 & \multicolumn{2}{|c|}{ A } \\
\hline M2 & 15.44 & \multicolumn{2}{|c|}{ A } \\
\hline M5 & 16.37 & \multicolumn{2}{|c|}{ A } \\
\hline M3 & 16.41 & \multicolumn{2}{|c|}{ A } \\
\hline T3 & 16.44 & \multicolumn{2}{|c|}{ A } \\
\hline T4 & 16.52 & \multicolumn{2}{|c|}{ A } \\
\hline M1 & 16.56 & \multicolumn{2}{|c|}{ A } \\
\hline M4 & 16.67 & \multicolumn{2}{|c|}{ A } \\
\hline T5 & 16.96 & \multirow[t]{6}{*}{ A } & B \\
\hline TOT1 & 29.89 & & B \\
\hline ТОT2 & 30.44 & & B \\
\hline ТОT3 & 32.85 & & B \\
\hline TOT4 & 33.19 & & B \\
\hline TOT5 & 33.33 & & B \\
\hline
\end{tabular}
que, a un nivel de confianza del $95 \%$, no existen diferencias significativas, en la frecuencia de opinión de los encuestados respecto a lugares y horas del día más ruidosos. 
Tabla 4. Medida de Sonido en los 5 puntos escogidos. Unidad decibeles (dB)

\begin{tabular}{|c|c|c|c|c|c|c|c|c|c|c|c|}
\hline \multirow[b]{2}{*}{ Mañana } & \multirow[b]{2}{*}{ Tarde } & \multicolumn{2}{|c|}{ OVALO } & \multicolumn{2}{|c|}{$\begin{array}{c}\text { SAN } \\
\text { IGNACIO }\end{array}$} & \multicolumn{2}{|c|}{$\begin{array}{c}\text { ESQ. } 28 \text { DE } \\
\text { JULIO Y } \\
\text { ECHENIQUE }\end{array}$} & \multicolumn{2}{|c|}{$\begin{array}{c}\text { PLAZUELA } \\
\text { SAN } \\
\text { MARTIN }\end{array}$} & \multicolumn{2}{|c|}{ UNJFSC } \\
\hline & & am & pm & am & pm & am & pm & am & pm & am & pm \\
\hline 7.30 & 1.30 & 80.1 & 82.1 & 78.3 & 80.1 & 79.5 & 79.1 & 78,9 & 80 & 78 & 80.7 \\
\hline 7.35 & 1.35 & 80.5 & 82.3 & 78.5 & 80.3 & 80.5 & 80.2 & 79 & 80.1 & 78.5 & 80.8 \\
\hline 7.40 & 1.40 & 80.8 & 83.1 & 78.7 & 80.2 & 80.6 & 80.1 & 79.1 & 80.1 & 78.8 & 80.9 \\
\hline 7.45 & 1.45 & 81.1 & 83.2 & 79.3 & 82.2 & 80.7 & 82 & 79.6 & 81.4 & 79.1 & 82.6 \\
\hline 7.50 & 1.50 & 89.8 & 85.1 & 79.8 & 83.7 & 80.8 & 83.1 & 79.8 & 82.9 & 79.8 & 83.6 \\
\hline 7.55 & 1.55 & 91.1 & 86.9 & 81.3 & 85.8 & 81.1 & 85.9 & 81.3 & 84.7 & 81.4 & 85.1 \\
\hline 8.00 & 2.00 & 90.6 & 85.8 & 80.7 & 84.9 & 80.9 & 84.7 & 81.7 & 84.9 & 80.7 & 84.8 \\
\hline 8.05 & 2.05 & 90.6 & 84.7 & 80.6 & 84.8 & 80.3 & 84.6 & 80.6 & 83.7 & 80.2 & 84.7 \\
\hline 8.10 & 2.10 & 86 & 83.9 & 80 & 83.7 & 79.2 & 83.4 & 80.4 & 83.6 & 80.1 & 83.5 \\
\hline 8.15 & 2.15 & 83,3 & 83.7 & 80,6 & 82.9 & 79 & 83.3 & 80,1 & 82.8 & 80,3 & 83.3 \\
\hline 8.20 & 2.20 & 81.3 & 83.4 & 81.9 & 82.4 & 78.9 & 83 & 79.4 & 82.4 & 81.9 & 82.5 \\
\hline
\end{tabular}

Tabla 5. MAÑANA: Medida de Sonido en los 5 puntos escogidos con sonómetro. Unidad decibeles $(d B)$

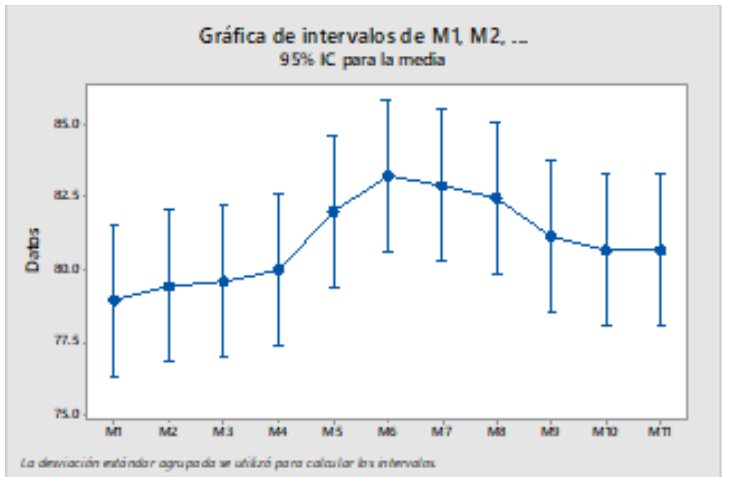

Tabla 6. TARDE: Medida de Sonido en los 5 puntos escogidos con sonómetro. Unidad decibeles $(d B)$

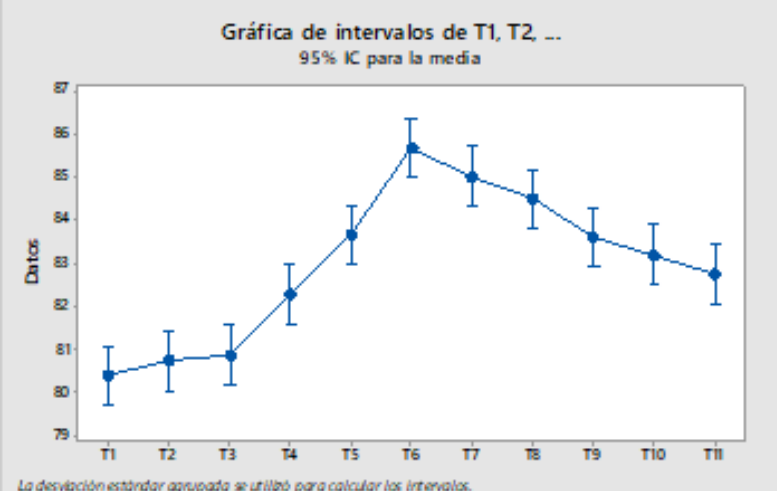

Big Bang Faustiniano 2018; 7(1):
Tabla 7. Análisis Para Lugares Y Horas De Medición

Medida de Sonido en los 5 puntos escogidos con sonómetro. Unidad decibeles (dB)

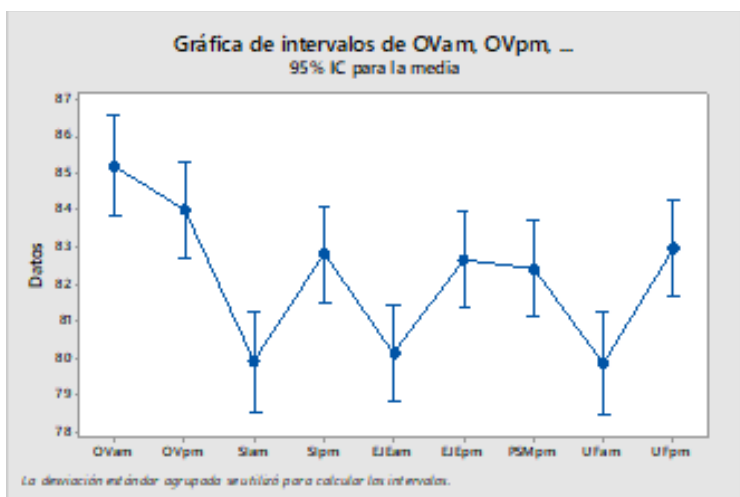

\section{DISCUSIÓN}

Los niveles de ruido urbano continúan aumentando a través de un mayor uso de las fuentes de ruido, una mayor extensión geográfica y la movilidad de dichas fuentes, así como la exposición a estos altos niveles es cada vezmayor (Torres 1998).

Existe una dificultad a la hora de asociar el efecto del sonido como una consecuencia aislada de otros factores ambientales y la discriminación de estos factores tiene especial relevancia cuando se plantea diseñar estudios de afección de ruido ambiente con respecto a las actividades de 
nuestra especie.

Hemos encontrado que los altos niveles de ruido producido por los claxon de los vehículos motorizados, generaron una respuesta adaptativa por parte de la mayoría de las personas que habitan, trabajan o transitan por las zonas de estudio. Los datos del estudio sugieren, que al producirse constantemente aumentos en los niveles de contaminación acústica, este progreso, podría afectar de una manera cada vez mayor a la comunicación entre las personas, pudiendo generar consecuencias en los patrones de conducta de la raza humana. (Henselman 1995)

El término "contaminación acústica" hace referencia al ruido (entendido como sonido excesivo y molesto), provocado por las actividades humanas (tráfico, industrias, locales de ocio, aviones, etc.), que produce efectos negativos sobre la salud auditiva, física y mental de los seres vivos. Este término está estrechamente relacionado con el ruido debido a que esta se da cuando el ruido es considerado como un contaminante, es decir, un sonido molesto que puede producir efectos nocivos fisiológicos y psicológicos para una persona o grupo de personas. (Ramírez 1998)

Organismos internacionales plantean que se corre el riesgo de una disminución importante en la capacidad auditiva, así como la posibilidad de trastornos que van desde lo psicológico (paranoia, perversión) hasta lo fisiológico por la excesiva exposición a la contaminación sónica. Un informe de la Organización Mundial de la Salud (OMS), considera los 70 decibeles (dB) (a), como el límite superior deseable, al superar estos niveles se pueden observar algunos efectos psicopatológicos y fisiológicos (Londoño 1997).

En comparación con Lima, se han registrado en Huacho, zonas con niveles de sonido de 91.1 decibeles, caso que está por encima de los 80 decibeles los cuales se puede percibir cuando un individuo está cerca de un tren de carga en movimiento, según lo señalado por la Organización Mundial de la Salud (OMS), superando ampliamente los 70 decibeles permitidos en esta zona de Lima, según el Organismo de Evaluación y Fiscalización Ambiental(OEFA).

En al menos 38 puntos de las ciudades de Lima y Callao, este nivel de sonido es ilegal. Por ejemplo, se exceden los niveles de sonido en avenidas como Javier Prado (78,8 db), la avenida Angélica Gamarra $(77,4 \mathrm{db})$, la avenida Elmer Faucett (79 db) y Morales Duárez (79 db), y también en el mercado Ceres de Ate $(80,3 \mathrm{db})$, en comparación con los puntos que se evaluaron en la zona de Huacho todos se encuentran superando el límite superior deseable que van desde los 78.9 a 91.1 decibeles, estando estos niveles de sonido por encima a los de las principales avenidas de la ciudad de Lima, lo cual nos indica la carencia del conocimiento por parte del sector de transporte de nuestra localidad sobre la contaminación acústica ya que en comparación el mayor el tráfico vehicular en Lima, la cantidad y el tipo de vehículos, nuestro sector presenta mayor contaminación acústica causada por una menor cantidad de vehículos. (Cabrera 2014)

La mayor parte de la gran contaminación sonora en las principales calles de las ciudades es causada principalmente por el uso indiscriminado de las bocinas en el tránsito vehicular", plantea Iván Pacheco Cajavilva, especialista de la Subgerencia de Medio Ambiente de la Municipalidad de Lima (MUNLIMA). El problema parece ir en aumento, señala Félix Cabrera Vega, analista en planificación de transporte y emisiones vehiculares, "definitivamente cada año aumenta el número de autos y la contaminación acústica", afirmó.

Hace varios años en las normativas de protección del ambiente no se consideraba el contaminante ruido, pese a que la industrialización y en si ciudades y países han ido creciendo y evolucionando, en todos los países del mundo se han elaborado normas y estatutos que se encargan de la rpotección del medio ambiente contra el exceso de ruido. Los esfuerzos mas serios de las comunidades internacionales se traducen en la profundización de los estudios sobre causas y origen (fuentes), deterioro y políticas de prevención y control de la contaminación sonora.

En España, se estableció como nivel de confort acústico los $55 \mathrm{~dB}$ por encima de este nivel, el sonido resulta pernicioso para el descanso y la comunicación. Según estudios de la Unión Europea (2005): «80 millones de personas están expuestas diariamente a niveles de ruido ambiental superiores a $65 \mathrm{~dB}$ y otros 170 millones, lo están a niveles entre 55-65 dB». 
(García 2006)

Directiva 2003/10/CE del Parlamento Europeo y del Consejo, de 6 de febrero de 2003, sobre las disposiciones mínimas de seguridad y de salud relativas a la exposición de los trabajadores a los riesgos derivados de los agentes físicos (ruido).

- Ley 37/2003, de 17 de noviembre, del Ruido.

- Real Decreto 286/2006, de 10 de marzo, sobre la protección de la salud y la seguridad de los trabajadores contra los riesgos relacionados con la exposición al ruido. ( Pérez 2000)

\section{CONCLUSION}

Observando los resultados de las mediciones in situ, se puede comprobar que los niveles de ruido emitidos por el tráfico rodado no cumplen con los límites establecidos.
Analizando los niveles con las dos franjas horarias, mañana y tarde, tal y como se indica en la normativa estatal, los puntos más conflictivos son en la franja de día, donde se presenta mayor tráfico en la mañana que por la tarde, teniendo las evaluaciones realizadas.

\section{AGRADECIMIENTO}

Al Blgo. Víctor Sánchez Cabrera, por su apoyo científico, revisión de la parte estadística, a la Lic. Moraima Yaniré Romero Canales, por su aporte en el desarrollo del presente trabajo, y a la Estudiante Yulexis Mayra del Carmen, Romero Canales, por apoyo en la redacción e impresión del presente, así mismo a los alumnos del II ciclo de Ingeniería Zootécnica, por su ayuda en la recolección de datos.

\section{REFERENCIA BIBLIOGRÁFICA}

Álvarez A. 1967 Ruido y Sordera. Contribución al estudio de la hipoacusia ocupacional. México: ed. El Nuevo Diario:12-7

Bosshardt HG, Hormann H. 1995. Protective inhibition: A central factor in auditory fatigue. Psychological research; 40:207-21

Cabrera Vega, Félix. 2013 Contaminación sonora en Lima provoca estrés y perjudica bienestar de la población. Visitado el 04 diciembre del 2014. Disponible en: http://tvperu.gob.pe/noticias/l o c a l e s / m e d $i$ o a mbiente/45045contaminacion-sonora-enlima-provoca-estres-yperjudica-bienestar-de-lapoblacion.html.

Eriksson-Mangold M, Carisson SG 1991. Psychological and somatic distress in relation to perceived hearing disability, hearing handicap and hearing measurement. Journal of Psychosomatic Research;
$35(6): 729-40$

García A. 1996 Medidas de niveles de contaminación acústica en diferentes zonas urbanas de España. TecniAcústica; 27(34): 7-21 en:

http://www.ia.csic.es/Sea/publicac iones/4350sr161.pdf

García A. 2006 Niveles de contaminación sonora en ciudades grandes, medias y pequeñas: un estudio comparativo. [en línea]. [27 de Julio del]. URL disponible

Henselman LW. 1995 Effects of noise exposore, race and yearsuf service on hearing in USArmy soldiers. Ear Hear; 16(4):382-91

Londoño JL, Restrepo H, Corrales AM, Mendoza F, Ortiz J. 1997 Hipoacusia neurosensorial por ruido industrial y solventes orgánicos en la gerencia Complejo Barrancabermeja, 1977-1997. Rev Nac Salud Pública;15(1):94-120

López AC, Fajardo GE, Chavolla R, Mondragón A, Robles MI. 2000 Hipoacusias por ruidos:
Un problema de salud $y$ de conciencia pública. Rev Fac MedUNAM;43(2):41-2

Pérez G. 2000 Protección de los trabajadores frente a los riesgos derivados de la exposición a ruidos. Mapfre Seguridad; 78:19-26

Portman M., Portman C. 1967 Audiometría Clínica. (TorayMasson, SA. Barcelona)

Ramirez R, López C. 1998 Alteraciones degenerativas cocleo vestibulares. En: Manual de Otorrinolaringología. Madrid: Mc Graw-Hill Interamericana: 141

Torres A., Pérez M. 1998Nuevas aplicaciones de la técnica de potenciales a múltiples frecuencias. En: Ministerio de Salud Pública. Recopilación de materiales del curso: "Audix" Nueva técnica para la evaluación objetiva de la audición. Ciudad de la Habana: Centro de Neurociencias de Cuba. 